\section{Cutaneous abscesses}

In Britain pyogenic infections of the skin and subcutaneous tissues account for about $5^{\circ}$ of all general practitioner consultations. ${ }^{1}$ In the past 30 years orthodox treatment has been based on the use of an increasing variety of antibiotics given topically or systemically. This approach is logical in the absence of frank suppuration, but it is highly questionable in patients with abscess formation. After comparative neglect for many years the important practical problem of how we should treat cutaneous abscesses has recently been clarified by Meislin $e t a l^{2}$ in Chicago. Such abscesses accounted for $2^{\circ}{ }_{0}$ of their adult emergency outpatient consultations, and they analysed 135 cases in detail.

Sensitive aerobic and anaerobic culture methods yielded bacteria from $96^{\circ}{ }^{\circ}$ of the abscesses in the series. Staphylococcus aureus was isolated from only $24^{\circ}{ }_{\mathrm{o}}$, but it was usually in pure culture, whereas other bacteria were mainly grown in mixtures. The most frequent staphylococcal lesions were in the axilla, while haemolytic streptococci were prominent only in hand infections. Interestingly, Proteus mirabilis (the commonest Gram-negative aerobe in the series) was implicated in most of the non-staphylococcal axillary abscesses. A possible explanation may be the popularity of under-arm deodorants, which tend to promote colonisation by proteus, whose biochemical activities may then damage the epidermis. ${ }^{3}$

As might be expected, anaerobes were present in most lesions situated between the thighs and waist, which constituted $40^{\circ}{ }_{0}$ of the abscesses. The predominant species belonged to the Peptococcus, Peptostreptococcus, Fusobacterium, and Bacteroides genera, though among the latter Bacteroides fragilis was less prevalent than penicillin-sensitive species. Nevertheless, the report's acceptance of an invariably pathogenic role for these miscellaneous bacteria was not apparently based on quantitative or semiquantitative data; and an even more doubtful assumption underlay the prominence given to lactobacilli and normal skin commensals in the culture results. This last group of bacteria may, indeed, often have a protective effect against true pathogens of the skin. ${ }^{4}$ Moreover, the unqualified statement that anaerobes, including peptococci, are normal residents of the human skin needs correction, since the only truly anaerobic commensals are believed to be the Propionibacterium group of "acne" bacilli (formerly Corynebacterium acnes).

The importance of the Chicago paper lies less in its varied bacteriological findings than in the fact that $74^{\circ}$ of the patients were cured of their abscesses by incision and drainage alone. The authors concluded that use of antibiotics should be restricted to high-risk patients, where there are signs of systemic infection, and in facial abscesses that may endanger the cavernous sinus. Their assertion that in all other patients "there is no indication for initial Gram-stain, culture, or antibiotic therapy" is, however, questionable. Drainage alone may not eradicate infection from the surrounding tissues, and bacteriological data-including antibiotic sensitivities-may prove valuable in immediate management or if there is recurrence. Furthermore, the doctor should not omit to test for glycosuria as part of his investigation.

Antibiotics are certainly not usually necessary as adjuncts to drainage when cutaneous abscesses are well localised. When their use is indicated-whether on the criteria of Meislin et al or because of substantial surrounding inflammation-antibacterial drugs should be chosen rationally. Co-trimoxazole may be inactivated by components of pus, ${ }^{6}$ while tetracyclines, erythromycin, fusidic acid, the lincomycin group, and amino- glycosides possess other disadvantages. Generally the penicillins or cephalosporins are preferable; but enthusiasm for cloxacillin or flucloxacillin should be tempered by the knowledge that both drugs (and their analogues) are highly protein-bound and penetrate relatively poorly into tissue and inflammatory fluids, ${ }^{7}$ and both are very vulnerable to destruction by penicillinases from bacteria other than Staph aureus. ${ }^{8}$ These drawbacks do not apply to some of the cephalosporins, notably cephradine, which is also the first of the group that may be given by mouth or injection. ${ }^{9}$ Nevertheless, the optimal use of this and other rapidly growing classes of antibiotics will depend increasingly on information derived from both bacteriology and the younger science of clinical pharmacokinetics.

${ }^{1}$ Office of Health Economics, paper No 46: Skin Disorders, p 11. London, 1973.

${ }^{2}$ Meislin, M W, et al, Annals of Internal Medicine, 1977, 87, 145.

${ }^{3}$ Shehadeh, $\mathrm{N} \mathrm{H}$, and Kligman, A M, fournal of Investigative Dermatology, 1963, 40,61.

* Selwyn, S, British fournal of Dermatology, 1975, 93, 487

5 Noble, W C, and Somerville, D A, Microbiology of Human Skin, Section Two. Philadelphia, Saunders, 1974.

${ }^{6}$ Lacey, R W, and Lewis, E, British Medical fournal, 1973, 4, 165.

'Craig, W A, and Welling, P G, Clinical Pharmacokinetics, 1977, 2, 252.

${ }^{8}$ Selwyn, S, fournal of Antimicrobial Chemotherapy, 1977, 3, 161.

${ }^{9}$ Kucers, A, Prescribers' fournal, 1977, 17, 109.

\section{Uncovering physical illness in elderly patients with dementia}

Both taking a history and performing a clinical examination may be difficult in patients with psychiatric illness. So Kampmeier ${ }^{1}$ has recently suggested that the traditional primacy given to the clinical history may not apply and that to make a diagnosis of physical illness certain diagnostic procedures and laboratory tests may assume an important role and be justified on the basis of an intuitive hunch or for screening.

Most of our elderly patients with organic psychiatric disorder still live in the community, ${ }^{2}$ and they form the most vulnerable section of the elderly resident community. When they require institutional care they take up beds mainly in residential medical geriatric and psychiatric units ${ }^{3}$ rather than in acute ones. Physical ill health is often said to be "silent" in these patients, but more often the fact is simply that the patient does not make any explicit or comprehensible complaints. Furthermore, doctors or nurses question their relatives and other informants too rarely. Assessing such a group of patients presents major problems, however, among the most important being the ethical ones. Should these patients be subjected to painful or even uncomfortable procedures or treatments when the downhill progress of most chronic organic psychiatric disorder renders the normal enjoyment of life unlikely? A less attractive but important question is how cost-effective is such medical investigation and treatment? What are we trying to prevent, and how may medical mismanagement create further problems that would be avoidable with better care?

Regrettably in Britain we do not have to ask the final question that Kampmeier poses: is the management of patients within the norms developed for quality care in general hospitals by professional standards review organisations? No 